\title{
SILICON-ON-INSULATOR-BASED OPTICAL ADD-DROP MULTIPLEXERS
}

\author{
Peter Gulvin, Joel Kubby, Jingkuang Chen, James Diehl, Kathleen Feinberg, Kristine German, Lawrence Herko, \\ Nancy Jia, Pinyen Lin, Xueyuan Liu, Jun Ma, John Meyers, Peter Nystrom, and Yao Rong Wang \\ Xerox Wilson Center for Research and Technology \\ Webster, New York 14580
}

\begin{abstract}
Optical Add-Drop Multiplexers (OADMs) are used for independently addressing the various wavelengths in wavelength division multiplexing (WDM) systems. Applications such as network restoration/reconfiguration, time-dependent bandwidth, and fiber to the home all require OADMs with good optical performance, small size, low cost, millisecond-range switching times, low power requirements, and good reliability over a variety of operating conditions.

Xerox has developed a single-chip integrated OADM built on a Silicon-On-Insulator (SOI) wafer that meets these requirements. The optical loss through a switch is less than $2 \mathrm{~dB}$. The entire system from fiber to fiber has $19 \mathrm{~dB}$ loss, an extinction ratio of over $40 \mathrm{~dB}$, and an adjacent crosstalk value of more than $30 \mathrm{~dB}$. Smoothing the waveguide sidewalls and decreasing input, output, and gap loss with anti-reflection (AR) coatings should decrease the loss to $12 \mathrm{~dB}$. The high optical index contrast between silicon and air allows for much tighter bending radii of the ridge waveguides than in similar silica-based approaches, reducing the chip size to only $1.5 \times 2 \mathrm{~cm}$ and decreasing the cost per device. The thermal driving and latching actuators switch within $12 \mathrm{~ms}$, using only 0.8 $\mathrm{W}$ of power, for a total of less than $10 \mathrm{~mJ}$ per change of state, with no power required to maintain the state. An unpackaged device ran for 17 million switch cycles without failure, and a hermetically-sealed device is expected to have even better reliability. Variable optical attenuators (VOAs) can be integrated with the devices for gain equalization without making any changes in the fabrication.

The devices can be fabricated using a manufacturing process developed through a National Institute of Standards (NIST) and Technology Advanced Technology Program (ATP) grant [1]. This process consists of a surface micromachining process done on top of an SOI wafer, allowing for guided as well as free-space optics, the integration of flip-chip bonded light sources, and the possibility of including integrated electronics. This broadly enabling process will allow fabrication of other applications in the same run, increasing volumes to be more cost-effective for the foundry customer.
\end{abstract}

\section{INTRODUCTION}

In WDM, multiple channels of information are encoded as different wavelengths. Independently handling these channels requires OADMs, which allow channels to enter or exit a WDM fiber. Currently OADMs cost $\$ 1,000$ per channel, restricting their use to long-haul communications. Decreasing the price per channel to $\$ 100$ would open them up to the metropolitan network level and applications such as network restoration and reconfiguration, time-dependent bandwidth, and fiber to the home [2].

To enable this $10 \mathrm{X}$ cost reduction of an OADM system, the system size was decreased by about 50X, and all the system components were integrated onto one chip [3]. To shrink the system, silicon waveguides were used instead of the more common silica waveguides. The higher optical index of silicon over silica leads to a minimum bending radius of $3 \mathrm{~mm}$, much smaller than the $10 \mathrm{~mm}$ minimum bending radius for a comparable silica waveguide. This allows the entire system to fit within a few square centimeters, as compared to the silica version that nearly fills a 6" wafer, cutting the cost by $50 \mathrm{X}$.

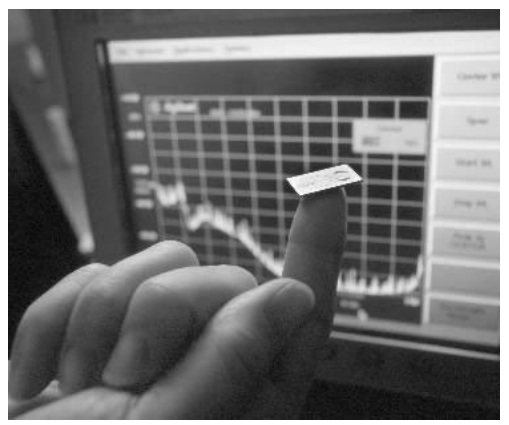

Figure 1. OADM chip on a fingertip

An OADM system requires a demultiplexer to separate the wavelengths, the switches to route the light to and from add/drop channels, and VOAs to level the output power of the channels. Typically each of these components is on a separate chip, and they are pieced together to create an OADM. This adds a great deal of cost and complexity to packaging. If all of these components could be created with the same fabrication process, then they could be integrated on one chip, saving space, cost, and potentially increasing performance by eliminating loss due to coupling between chips. Xerox's fabrication process is capable of producing the tapered input/output couplers for the fibers, the arrayed waveguide gratings (AWGs) used for multiplexing and demultiplexing, the optical switches, and the VOAs. As such, the process could be used for a wide range of optical systems in addition to OADMs, including $\boldsymbol{\lambda}$-routers and optical switches. The current prototype OADM includes the fiber couplers, AWGs, and switches, all on a $2 \times 1.5 \mathrm{~cm}$ chip.

The primary figures of merit for an OADM are optical insertion loss, extinction ratio, adjacent and non-adjacent crosstalk, power consumption, and reliability. While the current insertion loss of $19 \mathrm{~dB}$ is still too high, process development and antireflection coatings are expected to lower the loss to $12 \mathrm{~dB}$, which is competitive even when compared to much larger systems. The extinction and crosstalk values of 40 and $30 \mathrm{~dB}$ are already competitive, and should only improve as the fabrication process is optimized. The power consumption is $0.8 \mathrm{~W}$ during a change of state, and the $12 \mathrm{~ms}$ duration of a state change means that it consumes less than $10 \mathrm{~mJ}$. No power is necessary to maintain the state, giving the additional benefit that the switches maintain their state during a power outage. Also, the small size of the system greatly reduces the energy required to maintain its temperature. The unpackaged switches have been run for more than 17 million cycles without failure, and hermetically-sealed packaged die should have increased lifetime. 


\section{THEORY}

\section{Ridge Waveguides:}

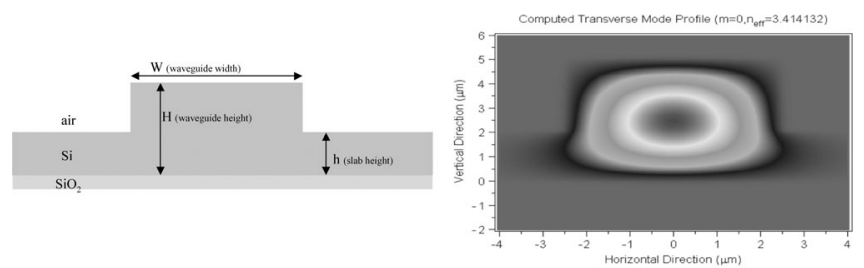

Figure 2. Ridge waveguide and its transverse mode profile [2]

An optical fiber guides light by confining it to a high index region (core) surrounded by a lower index material (cladding). A ridge waveguide is similar, except the index differentiation is achieved by changing the material's thickness, as shown in Fig. 2 .

To fully understand a ridge waveguide one would have to solve Maxwell's equations for the different thickness regions and match the solutions at the boundaries to determine the allowable eigenmodes. However, one can use the effective index method to determine the effective index of the ridge (larger thickness) region and the slab (smaller thickness) region. In the ridge region the light is mostly confined to the silicon, and thus the index is that of the silicon $(n=3.5)$. In the slab region a larger percentage of the light is in the air, and the resulting effective index is a weighted average between that of the silicon $(n=3.5)$ and that of the air $(n=$ 1). To find the actual value modeling software is used to analyze the mode shape and determine the effective index. The example in figure 3 uses BeamPROP ${ }^{1}$ optical modeling software to analyze a 5-micron thick, 5-micron wide ridge region and 2-micron thick, infinitely wide slab region. The result is shown as $n_{\text {eff }}=3.414$.

\section{Arrayed Waveguide Gratings:}

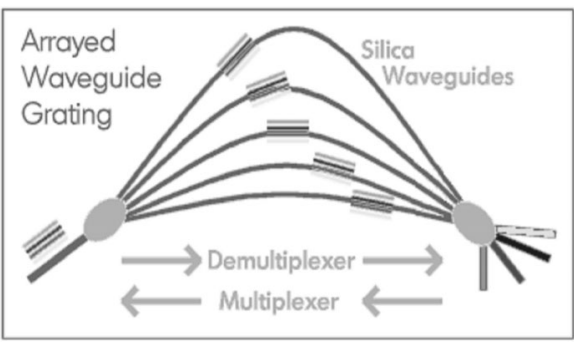

Figure 3. Conceptual drawing of $A W G$ (www.lightreading.com)

An arrayed waveguide grating (AWG) uses interference between a large array of waveguides to direct different wavelengths of light to different output channels. When an AWG is acting as a demultiplexer, light enters from a single waveguide and fans out in a region called the "star coupler". The light then enters an array of intermediate waveguides, each of a slightly different length and each carrying part of all the wavelength channels. As a particular wavelength exits the intermediate waveguides, it interferes with the same wavelength exiting the other intermediate waveguides, and is sent through the exit star coupler toward the output waveguides. The output waveguide for a particular wavelength channel is placed at the point where that wavelength has its maximum constructive interference. Thus each wavelength channel is aimed at the proper output waveguide.

\footnotetext{
${ }^{1}$ BeamPROP is produced by RSoft Inc, www.rsoftdesign.com/
}

Increasing the number of intermediate waveguides will strengthen the interference, leading to a more well-aimed, well-defined spot, but at the cost of a larger AWG.

The same process can be turned around, sending light in what were previously output channels, combining them, and then sending them multiplexed together in one waveguide.

\section{Thermal Actuators:}

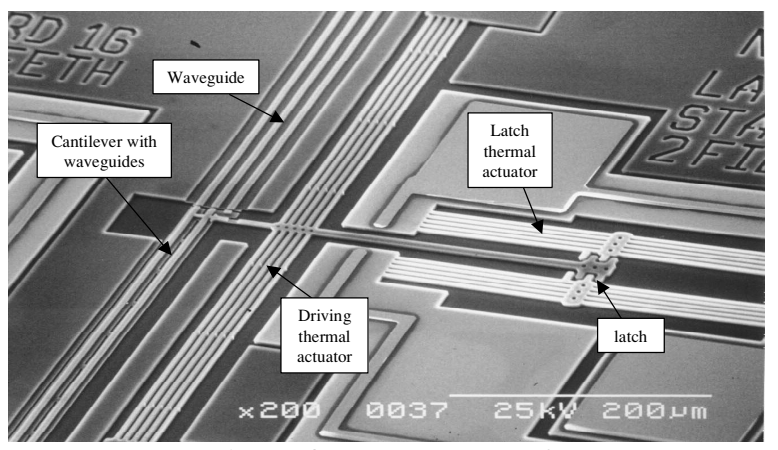

Figure 4. OADM system [2]

Thermal actuators in general use electric current to cause Joule heating, and the resulting thermal expansion causes motion. A straight beam between two anchors would buckle unpredictably when heated, so typically the center of the beam is offset slightly to one side to assure motion in that direction. This is known as a "Vbeam" actuator.

When a v-beam actuator is subjected to film stress (e.g.: doped silicon or polysilicon), it will either bend more or straighten, depending on whether the stress is compressive or tensile respectively. In an optical switch where one of the states is the asfabricated position, the displacement that results from stress is highly undesirable.

To eliminate this effect, methods besides an offset must be used to break the symmetry and bias the motion of the actuator in the desired direction. One possibility is to vary the spacing of the beams to alter their rates of heating and cooling, and thus their ultimate temperature. Another is to vary the doping levels of the beams to alter the Joule heating [2]. In either case beams on one side heat more and at a quicker rate than those on the other, and the actuator moves in the direction of the beam that expands the most or the most quickly.

Another challenge with thermal actuators, particularly with longer, higher displacement actuators, is that the voltage difference between the beams and the floor beneath them can cause the beams to electrostatically pull down and stick to the floor, causing device failure. To minimize this, the voltage applied to the two ends is not single-ended, but rather bipolar. Thus the center of the beams are at zero potential, and the downward force is minimized (other areas along the length still have a downward force). Another way to minimize this effect is to more heavily dope the beams, decreasing the voltage required to achieve the same current. Since a higher doping level leads to higher stress levels, this further increases the need for a design that doesn't displace easily due to stress. 


\section{EXPERIMENTAL DETAILS}

Fabrication process:

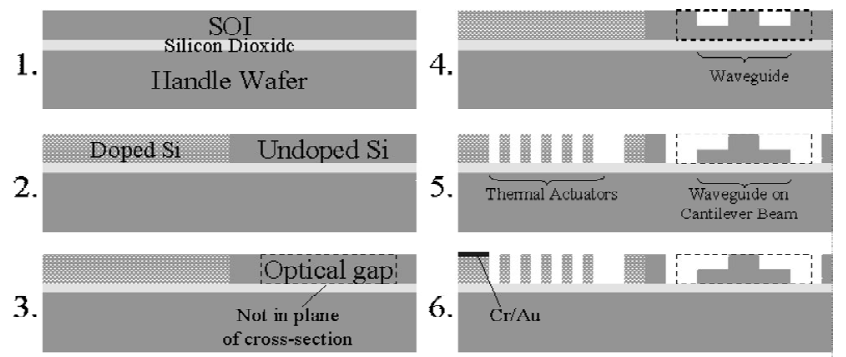

Figure 5. OADM fabrication process

1. Starting wafer: The process begins with an SOI wafer with a 5 or 6 micron device layer, and a 1 or 2 micron buried oxide layer.

2. Patterned doping: This provides greater conductivity for the thermal actuators to lower the voltage requirements, while avoiding doping of waveguides to prevent absorption of photons.

3. Critical gap silicon etch: This etch defines the optical gap between the moving and stationary sides of the optical switch, since the resulting Fabry-Perot cavity will have significant loss unless it is tuned to a multiple of half the wavelength or if AR coatings are applied. To get as close as possible to an optimal value the lithography must be very accurate, and the flat silicon at this stage will help.

4. Waveguide etch: This timed partial-depth silicon etch defines the ridge and slab regions of the waveguides.

5. Mechanical silicon etch: This etches all the way through the silicon, stopping on the buried oxide layer. The thermal actuators, cantilever beams, latch beams, traces, and bond pads are defined at this step.

6. Patterned metal: Chrome/Gold is patterned using a lift-off process, coating the bond pads and optionally the traces.

\section{Switch Operation:}

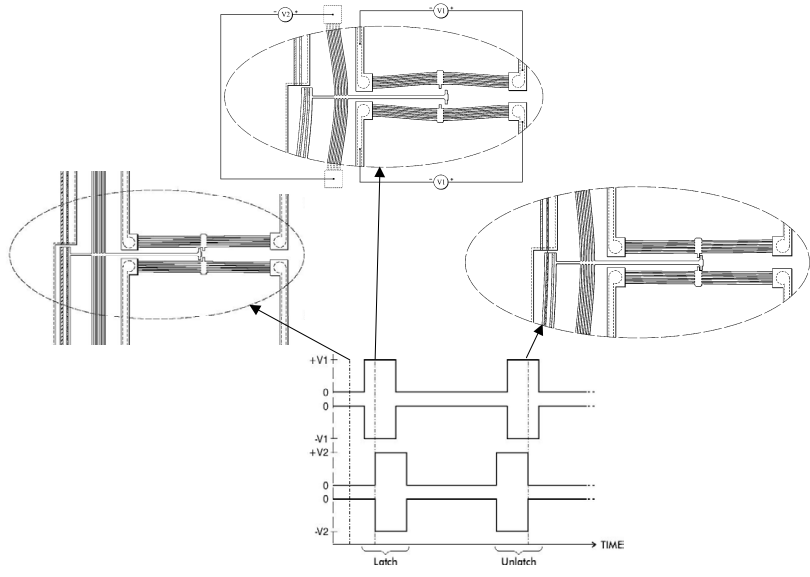

Figure 6. Diagrams of unlatched, intermediate, and latched states, and a plot of voltage vs. time [2]

Shown above is a diagram of how the devices are latched and unlatched. To latch the device the latch actuators are opened, the drive actuator moves the waveguides so that the latch teeth are beyond where they need to be, the latch actuators close, and the drive actuator relaxes so that the waveguides settle into their latched state. To unlatch the device, the drive actuator is again actuated to separate the latch teeth and prevent friction from causing binding, the latch actuators open, the drive actuator relaxes and moves the waveguides to their original state, and the latch actuator closes. Figure 7 shows what the waveguides look like in their unlatched and latched states.
Add/Drop State

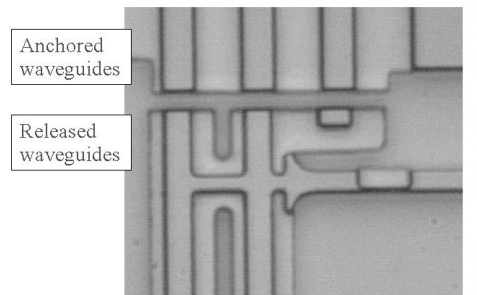

Figure 7. Close-up view of waveguides in both states[2]
Unpackaged devices have been successfully run for over 17 million latch/unlatch cycles without seeing a failure or any significant drifting in the positions of the states. Some oxidation was evident near the midpoints of the drive actuators, where the maximum temperature is achieved. By hermetically sealing the devices in an inert gas, the oxidation should be eliminated. The devices were left in their latched states for weeks to test whether stiction in the latch teeth might pose a problem, but they started right up without a problem.

\section{Optical Testing:}

To test the devices, lensed fibers were used to couple broadband illumination into a waveguide that is tapered in the plane of the chip (no thickness variation). To determine the loss at each wavelength, the spectrum of the input and output light was measured and the difference in power at each wavelength was noted.
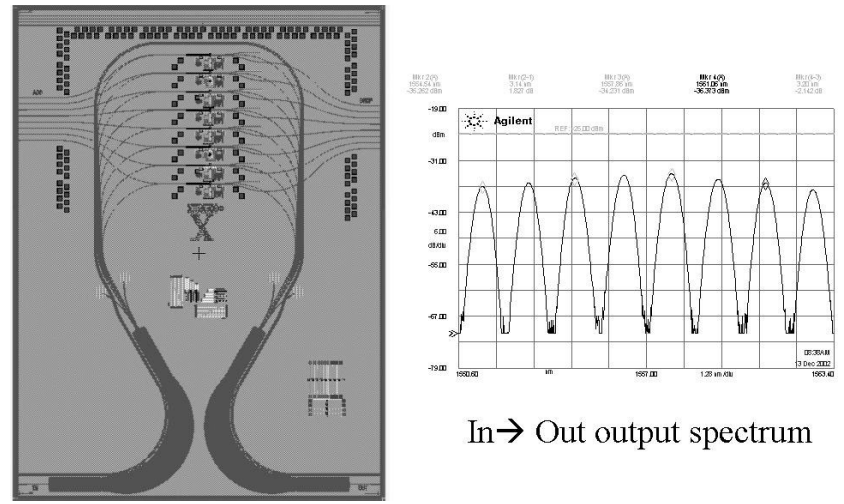

In $\rightarrow$ Out output spectrum

Figure 8. Example design and the resulting output spectrum

The insertion loss for an entire system was measured to be $19 \mathrm{~dB}$. To determine the loss breakdown by component, a series of test structures were used, such as AWGs by themselves, switches with waveguides, straight waveguides, and curved waveguides. The table below lists the losses that were found, the degree to which the loss can be improved, and the method by which the improvement will occur. The numbers don't add to $19 \mathrm{~dB}$ since the parts used to measure component losses were not from the exact system that the $19 \mathrm{~dB}$ figure came from: 


\begin{tabular}{|l|c|c|l|}
\hline \multicolumn{1}{|c|}{ Source of Loss } & Current IL & Target IL & \multicolumn{1}{c|}{ Improvement Method } \\
\hline Fiber-WG coupling & $4.7 \mathrm{~dB}$ & $<2 \mathrm{~dB}$ & Add AR coating \\
\hline Waveguide Loss $(5.3 \mathrm{~cm})$ & $4.3 \mathrm{~dB}$ & $<2.5 \mathrm{~dB}$ & Sidewall smoothing \\
\hline 2 x AWG & $8 \mathrm{~dB}$ & $6 \mathrm{~dB}$ & Sidewall smoothing/Design Optimization \\
\hline Waveguide Gap & $2 \mathrm{~dB}$ & $1 \mathrm{~dB}$ & Add AR coating \\
\hline Crossings, S-bends, etc. & $1 \mathrm{~dB}$ & $1 \mathrm{~dB}$ & None \\
\hline Total & $\mathbf{2 0 d B}$ & $<\mathbf{1 2 d B}$ & \multicolumn{2}{|c|}{}
\end{tabular}

Figure 9. Table analyzing optical losses

When an AR coating is added to the waveguide gap, the Fresnel loss in the gap will be eliminated and tight control of the gap width will not be necessary. That will allow the waveguide gap to be etched at the same time as the rest of the mechanical features, saving one mask and a few processing steps and making the process much more manufacturable.

The need for sidewall smoothing is due to vertical striations and other roughness in the reactive ion etching (see figure 10).

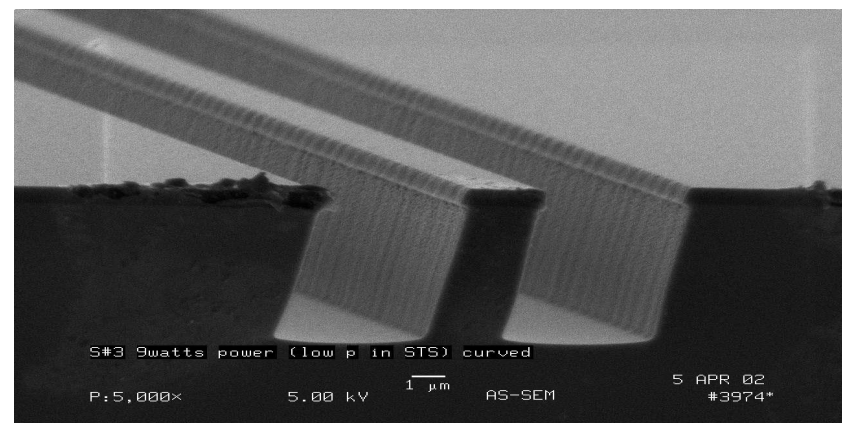

Figure 10. Etched features showing vertical striations

\section{Manufacturing Process:}

The fabrication process described above is a custom process designed solely to make the OADM. Versions of the device have been made using a manufacturing process developed through an Advanced Technology Program (ATP) grant from the National Institute of Standards and Technology [1]. This process consists of a surface micromachining process done atop an SOI wafer, allowing for guided as well as free-space optics, the integration of flip-chip bonded light sources, and the possibility of including integrated electronics. This flexible process will allow fabrication of other applications in the same run, increasing volumes to be more cost-effective for the foundry customer.

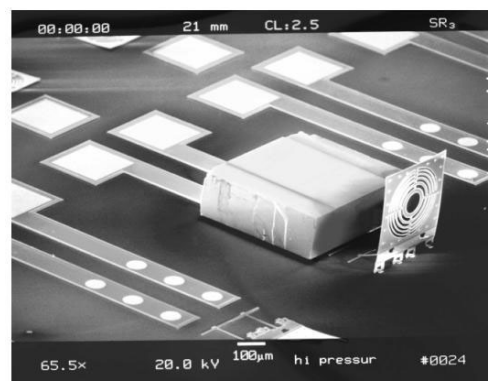

Figure 11. Flip-chip bonded laser diode and fresnel lens made with ATP process [4]

There are plans to transfer this process developed under an ATP grant to the Infotonics Technology Center in Canandaigua, NY within the year. The Infotonics Center was created with the intent of sharing infrastructure that would be prohibitively expensive and underutilized were one company to purchase it on their own, and the ATP process will be one of the first process qualified in the Center.

\section{CONCLUSIONS}

An OADM has been created that performs nearly as well as much larger systems, but with substantial size and cost advantages. With further process development and some work with AR coatings, the OADM should be able to be improved to the point where it is competitive with other OADMs in any cost or size range.

A fabrication process has been created that is capable of making much more than just OADMs. A $\lambda$-router has also been demonstrated [2], and has been shown to function as expected, with extra loss due to the additional pair of AWGs. Other applications such as optical switching may also be possible using the same process.

\section{ACKNOWLEDGEMENTS}

Process work performed under Cooperative Agreement \#70NANB8H4014, National Institute for Standards and Technology Advanced Technology Program, ATP Project Managers Thomas Lettieri and Carlos Grinspon.

\section{REFERENCES}

1. J.A. Kubby on behalf of the MOEMS Manufacturing Consortium, "Combining light with motion: hybrid integration of light emitters and detectors with SOI-based micro-opto-electro-mechanical (MOEMS) systems", Proceedings of SPIE, 4293, 32-45 (2001).

2. K. German et al., "Optical MEMS platform for low cost on-chip integration of planar light circuits and optical switching", Submitted to Proceedings of SPIE, 5357, 2004

3. J.A. Kubby, "Advances in MEMS integration with planar waveguide devices", Proceedings of SPIE, 4654, 1-12 (2002).

4. P. Gulvin, "Hybrid Silicon-On-Insulator Micromachining Process: 8 Mask Version Design Guide”, 2004 www.infotonics.org/Newsroom/documents/scssmmv2.pdf 